Please quote as: Köbler, F.; Leimeister, J. M. \& Lugmayr, A. (2006): Framework for Systematic and Strategic Planning of Innovation-based Mobile Broadband Services. In: 14th Euromicro International Conference on Parallel, Distributed, and NetworkBased Processing (PDP06), Washington, DC, USA. 


\section{Framework for Systematic and Strategic Planning of Innovation-based Mobile Broadband Services}

\author{
Felix Köbler \\ University of Technology \\ Munich, Department of \\ Informatics, Business \\ Informatics, \\ koeblerf@in.tum.de
}

\author{
Jan Marco Leimeister \\ University of Technology \\ Munich, Department of \\ Informatics, Business \\ Informatics, \\ leimeister@in.tum.de
}

\author{
Artur Lugmayr \\ Tampere University of \\ Technology, Inst. for Signal \\ Processing, New Ambient \\ MUltimedia (NAMU) Group, \\ lartur@acm.org
}

\begin{abstract}
The underlying paper describes a framework for the systematic and strategic planning of mobile broadband services, providing an industrial application and an impulse for further and future research on adoption and diffusion studies in the field of mobile broadband technology.

Elaborated conditions and characteristics of innovations, keyfindings of basic theoretical approaches on adoption and diffusion studies and the critical review of advanced and recently developed modelling approaches are combined with the special characteristics of the telecommunication industry, to a two-level based framework describing factors of influence on adoption and diffusion of mobile broadband services.

The framework strength is in its detailed state-of-theart description of current industrial mobile broadband situation and the two-level - micro- and macro-level approach on adoption and diffusion research. The novelty of the framework is its potential exemplary appliance on Germany, Finland or other countries, extraction of key-patterns in this highly competitive business field, highly consumer-oriented approach and its advanced scopes and recommendations for mobile broadband service development units.
\end{abstract}

\section{Introduction}

The paper originates out of a Bachelor Thesis (Bachelor of Science), the keyfindings are elaborated out of an in-depth analysis and description of mobile telephone and mobile standard evolution with concentration on countries embedded in the European Union and special descriptions of the historical and current market structure evolutions in Finland and Germany. Followed by a critical review and discussion of principal and process descriptions, frameworks and models, and current approaches and evolutions in adoption and diffusion research applied to the mobile technology sector. The synthesis of characteristics of innovations and implifications in the adoption and diffusion process and its influencing factors on the mobile broadband market from screened literature lead to a framework enabling strategic planning of mobile broadband services, in a two-level approach. The macro-level describes the social system through attributes, in which the innovative mobile broadband services are introduced. The micro-level describes the perceived factors on an individual level in bundled categories deduced from the previous analysis.

\section{Mobile broadband services}

Mobile broadband services are part of the mobile telephone evolution and embedded in the mobile standard evolution process, driven by highly sophisticated transmission and mobile technology developments, like the third (3G) or fourth (4G) generation of mobile telecommunication, disruptive technolgies like wireless LAN or WiMAX (Worldwide Interoperability for Microwave Access) [1] or new networking concepts like pervasive networks, mobile mesh network [2] and ambient networking [3]. Stated key-developments push the door open to an innovation-orientated service-dominated phase of mobile telecommunication on the providing side and 
result in complex acceptance and usage behaviour of customers.

A sophisticated approach on emerging mobile broadband services is presented by the scholars Varshney/Vetter [4] including a proposed classification of services, whereas there is a need to advance the framework and classification away from a pure commercial alignment to a broader spectrum of services. For the underlying framework mobile broadband services are classified as an unit or a bundle of information systems (IS), describable as a conglomeration of human, machine and methods units, interacting and being connected to execute specific functions [5] and social consumer-orientated systems with an emphasis on business-to-consumer and consumer-to-consumer services, e.g. group-related IS and virtual mobile communities. Mobile broadband IS are communication- and information systems, as information is communicated and computed through various channels and feature highly mobile, real-time and possible location-based information to serve a user unit embedded in its mobile environment.

\section{Adoption and diffusion studies}

The analysis of exisiting base and mobile telecommunication amplified adoption and diffusion models base on an innovation-centered driven perspective considering the perception of innovation by Drucker [6] exposing a set of three influencing entities: technology, innovation and entrepreneurship; resultant in sources for innovative opportunity.

This given presupposition makes it reasonable to imply theoretical- and practical-elaborated findings of exante or/and ex-post, adoption and diffusion data and model patterns, combined with influential developments of potential innovations of today's telecom sector.

The elaborated framework bases on traditional adoption and diffusion modelling approaches, their analysis and critical review, such as the Innovation Diffusion Theory (IDT) according to Rogers [7] and the Technology-Acceptance Model (TAM) according to Davis [8]. Furthermore two chosen wireless-focused amplification models are presented, discussed and critically reviewed.

The analysis focused on the principles and structure of the Wireless Technology Diffusion Model presented by Gera/Chen [9], which tends two objectives: to assist the stakeholders in analyzing the "potential success/failure of their new wireless applications or services prior to their launch" and in planning of their "strategies for the entire diffusion process" [9]. The lack of socio-logical focus of the IDT is balanced with the adoption of components of the TAM. The amplified model is a hybrid of constructs and applied on wireless technology services, but it is not focusing on special characteristics of the technology and services itselves.

The second analysed amplified model is the Trust Enhanced Technology Acceptance Model (TOMI), an amplification of the TAM, focusing on explaining the use of mobile payment solutions, according to the analysis of the scientists Dahlberg, Mallat \& Öörni [10] emphasizing on the influencing factor trust towards IS service solutions. Point of criticsm is the subdivisional view of the model and the emphasis on special personal characteristics which therefore leads to a loss of inherited commonality and integrity of its base model.

\section{Framework description}

In imitation to the categorisation of adoption and diffusion models by Frank [11] in micro- and macrolevel models, the framework tries to counteract the fact of high specialisation (micro-level orientated models) and generalisation (macro-level orientated models) with a two-level approach and a manageable amount of screened attributes. In short, the micro-level discribtion of perceived attributes control the adoption decision process of individuals or decision-making units, and the macro-level conditions describe the social system adoptional units (in particular innovators, early adopters and early majority) are embedded in.

\subsection{Macro-level: social system "attributes"}

The macro-level approach on the adoption and diffusion of mobile broadband services tries to define constructs of the social system in deducing factors (introduced as attributes) which influence the individual or decision-making units and describe the range of underlying conditions. An individual or decision-making unit is embedded in a social system, which is defined through so-called dimensions forming the environmental space. In this environmental space adoption decisions are taken and diffusion of mobile 
broadband services and other technologies take place, in a dynamic interplay of attributes. In this work four different types of dimensions are identified: the (1) cultural and demographic, (2) technological, (3) economical and (4) political and jurisdictional dimension. A dimension is defined through attributes (refer to object-otientated methodology) that describe the character of each dimension and its influence on the environmental space. The attributes can be regarded as external variables that take an indirect effect on embedded technology service solutions, and therefore carry importance on the systematical and strategical introduction of mobile broadband services. It should be anoted that dimensions can influence each other; changes of attributes in one dimension can affect the whole system, e.g. jurisdictional decisions can cause changes in the technological dimension.

One assumption $[12,13,14,15,16]$ of the framework is the growing influence of cultural and demographical-defined dependent and non-dependent attributes: individualism, collectivism, low-context, high-context [17], uncertainty avoidance, power distance, avertiveness [13]; spanning the cultural and demographic dimension. The technological dimension depicts macro influences on mobile broadband services from a technological background, described through: degree of interconnectivity [18] with other parallel or previous emerging technologies, degree of standardization [19], facilitating conditions [12, 19], facilitating conditions [19] in the evolution process of a technology. Following attributes for the economical dimension are deriveable from literature: demand attribute [19], cost conditions, the competitive rivalry [19] of mobile application developer, content provider, service provider, handheld developers and manufacturers, network technology developers and operating system developers, market size, general economical situation, suitabale cooperation possibilities [18], transferability conditions [19] and the activity of consumers. The political and jurisdictional dimension describes firstly the influence of politic decisions on the implementation rules of mobile technology infrastructure and market regulations with implifications for mobile broadband services and secondly judgments from influential jurisdictional units through elaborated attributes: effect of regulations, socio-economical effects that include consequences on restrictions, limitation of usage and protection of data privacy.

\subsection{Micro-level: perceived attributes of adoptional units}

The second iteration of the framework is the microlevel [15] describing the direct effect on individual adoption units cause by micro-level dimensions: (1) innovational , (2) technology, (3) marketing and (4) social.The innovational dimension carries following influencive attributes deduced from characteristic of an invention: market-pull or technology-push introduction strategies, general degree of knowledg, perceived improvement and novelty [6], degree of incongruity [6] and perceived diversity. Technology perceived attributes are the characteristics of an implemented innovative service solution in the actual interaction process of individual adopter units and defined through the following elaborated attributes: complexity of usage [6], relative advantage compared to parallel introduced service solutions, security and trust and connectivity. Through the dimension of marketingrelated attributes: pricing, promotion and advertising and distribution availability [20], the future user or current user gets informed by marketing channels about new technological developments in the form of market-introduced services. The framework assumes two main attributes that describe social perceived characteristics of a mobile broadband service solution, servicing two social needs of the user: socialising and entertainment [12].

An in-detail description of all underlying dimensions (macro- and micro-level) and listed attributes please refer to the underlying thesis, whereas correlations and emerging effects between each dimensional layer are not part of the framework, as a description would flow into a model design.

\section{Conclusion}

The framework tries to give a new impulse (balanced approach in detail and generality) on adoption and diffusion studies (clearly differentiating between both of them) related to recent eveloving technologies, providing a new theoretical approach applicable for industrial units to analyse research, development and managerial processes and actions. Further work aims on the inclusion of practiceorientated information (underlying thesis' questionnaire) to develop a model description (weighted influence of attributes) of the adoption and diffusion process of mobile broadband services. 
The macro-level description can be applied on countries, cutural- defined areas and economic regions. (Please refer to underlying thesis) The micro-level application aims on the description of design recommendations and managerial implications to control the attributes that influences and take effect on the adoptional unit; like market- and situationalaligned strategies (including e.g. certain launch waiting period), low degree of complexity implementations (e.g. well-structured menus), focus group adjusted promotion and advertising activities and focused socialising solutions (e.g. focus on group-related location-based services) and combined entertainment perceived use activities.

\section{References}

[1] Anonymous/Electronic Source, "Encyclopedia: 4G", http://www.nationmaster.com/encyclopedia/4G, accessed 05.07.2005, 2005.

[2] Anonymous/Electronic Source, "Mobile Mesh Networking : extending the dumb network." http://mcabiling.persozone.net/mobilemesh/mobilemesh.html , accessed 05.07.2005, 2004.

[3] Anonymous/Electronic Source, "Ambient Networks", http://www.ambient-networks.org/index.html, accessed 05.07.2005, 2005.

[4] Varshney, U.; Vetter, R., "A Framework for the Emerging Mobile Commerce Applications", Paper presented at the 34th Hawaii International Conference on System Sciences, Hawaii, U.S.A., 2001

[5] Davis, W.S., "Systems Analysis and Design - A structural Approach", Addison-Wesley, 1983.

[6] Drucker, P.F., "Innovation and Entrepreneurship", Butterworth-Heinemann, 2001.

[7] Rogers, E.M., "Diffusion of Innovations", The Free Press, New York, 2003.

[8] Davis, F.D., “A Technology Acceptance Model for Empirically Testing New End-User Information Systems: Theory and Results", MIT Sloan School of Management, 1986.

[9] Gera, K.; Chen, L. (2003), "Wireless Technology Diffusion: An evaluation model for wireless services", Paper presented at the Ninth Americas Conference on Information Systems, 2003, pp. 1961-1974.

[10] Dahlberg, T.; Mallat, N.; Öörni, A., "Trust Enhanced Technology Acceptance Model - Consumer Acceptance of Mobile Payment Solutions", Helsinki School of Economics, 2003.

[11] Frank, L.,'Mobile Communications within the European Union: The role of Location in the Evolution and Forecasting of the Diffusion Process", Lappeenranta, Finland, 2003.

[12] Pedersen, P.E.; Ling, R., "Modifying adoption research for mobile Internet service adoption: Crossdisciplinary interactions", Paper presented at the 36th Hawaii International Conference on System Sciences, Hawaii, U.S.A., 2002, p. 1-10.

[13] Straub, D.; Keil, M.; Brenner, W., "Testing the Technology Acceptance Model across Cultures: A Three Country Study", In: Information \& Management 31, 1997, p. $1-11$.

[14] Ives, B.; Jarvenpaa, S., "Wiring the Stateless Corporation: Empowering the Drivers and Overcoming the Barriers", In: SIM Network (6:5), 1991, p. 5-8.

[15] Frank, L., "Adoption of Mobile Services in Finland, Germany and Greece", Lappeenranta University of Technology, 2001.

[16] Frank, L.; Sundqvist, S.; Puumalainen, K.; Taalikka, S., "Cross-Cultural Comparison of Innovators: Empirical Evidence from Wireless Services in Finland, Germany and Greece", Lappeenranta University of Technology, 2001.

[17] Gudykunst, W.B.; Ting-Toomey, S., "Culture and Interpersonal Communication”, In. Sage Publications. Sage Publications, 1988, p. 39-53.

[18] Vassiopoulou, K.; Ziouvelou, P.; Pateli, A.; Pouloudi, A., "Examining E-Business Models: Applying a Holistic Approach in the Mobile Environment", Paper presented at the 11th European Conference on Information Systems (ECIS), Naples, Italy, 2003.

[19] Lehrer, M.; Dholakia, N.; Kshetri, N., „National Sources of Leadership in $3 \mathrm{G}$ M-Business Applications: A Framework and evidence from three global regions", Paper presented at the Eighth Americas Conference on Information Systems, 2002, p. 1825-1828.

[20] Puumalainen, K., „Global Diffusion of innovations in telecommunications: Effects of data aggregation and market environment", Lappeenranta, 2002. 